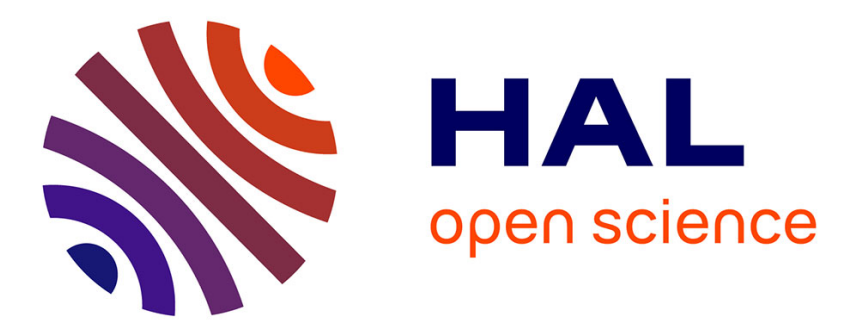

\title{
Analyse de la douleur urétrale lors de la pratique de l'autosondage: étude rétrospective sur 77 patients
}

N. Turmel, C. Hentzen, G. Miget, C. Chesnel, A. Charlanes, F. Le Breton, E. Tan, G. Amarenco

\section{- To cite this version:}

N. Turmel, C. Hentzen, G. Miget, C. Chesnel, A. Charlanes, et al.. Analyse de la douleur urétrale lors de la pratique de l'autosondage: étude rétrospective sur 77 patients. Progrès en Urologie, 2019, 29, pp.360 - 365. 10.1016/j.purol.2019.04.005 . hal-03484397

\section{HAL Id: hal-03484397 https://hal.science/hal-03484397}

Submitted on 20 Dec 2021

HAL is a multi-disciplinary open access archive for the deposit and dissemination of scientific research documents, whether they are published or not. The documents may come from teaching and research institutions in France or abroad, or from public or private research centers.
L'archive ouverte pluridisciplinaire HAL, est destinée au dépôt et à la diffusion de documents scientifiques de niveau recherche, publiés ou non, émanant des établissements d'enseignement et de recherche français ou étrangers, des laboratoires publics ou privés.

\section{(ㄷ)(1) $\$$}

Distributed under a Creative Commons Attribution - NonCommerciall 4.0 International 


\section{Titre et auteurs}

Analyse de la douleur uretrale lors de la pratique de l'autosondage : étude rétrospective sur 77 patients

N. Turmel, C. Hentzen, G. Miget, C. Chesnel, A. Charlanes, F. Le Breton, E. Tan, G. Amarenco.

Sorbonne Université, GRC 001, GREEN Groupe de Recherche Clinique en Neuro-Urologie, APHP, Hôpital Tenon, F-75020 Paris, France

Auteur correspondant : Nicolas TURMEL

nicolas.turmel@aphp.fr 


\section{INTRODUCTION}

Les autosondages intermittents sont le traitement de référence de la rétention urinaire chronique, principalement dans la population des patients neurologiques $[1,2]$. Cette rétention chronique peut être liée à plusieurs éléments étiopathogéniques tels qu'une hypocontractilité détrusorienne (secondaire à une pathologie neurologique ou mécanique telle que les mégavessies congénitales, les detrusors claqués ou pharmaco induite), une dyssynergie vésico sphinctérienne (neurologique ou fonctionnelle) ou encore un obstacle sous vésical [3]. Le traitement de cette rétention est bien souvent indispensable afin d'éviter tout risque sur le haut appareil, toute infection récurrente et tout symptôme directement lié à la mauvaise vidange vésicale (pollakiurie, fuites par regorgement). Bien que l'autosondage ait un impact positif sur la qualité de vie [4] (soit par amélioration directe des symptômes soit par la possibilité d'associer alors des thérapeutiques déprimant l'excès d'activité vésicale), certains patients décrivent des douleurs lors de la réalisation de l'autosondage pouvant pénaliser adhérence et compliance au traitement. Dans une étude sur des blessés médullaires, Yilmaz et al. [5] retrouvaient que 30,2\% des patients craignaient de ressentir des douleurs lors du sondage. La douleur ou les sensations de blocage peuvent mener à une adhésion faible aux autosondages et ce notamment dans une population de patients neurologiques avec préservation de la sensibilité urétrale et plusieurs traitements associés [6]. Cependant peu d'études sont disponibles sur la fréquence de ces phénomènes douloureux lors des autosondages.

L'objectif de cette étude était de déterminer la proportion de patients ayant des douleurs lors de l'autosondage et d'en vérifier l'impact en termes d'adhérence au traitement.

\section{METHODES}


Nous avons réalisé une étude rétrospective monocentrique sur des patients admis dans un service de Neuro urologie pour apprentissage de l'autosondage entre Janvier 2018 et Mars 2019. Les patients inclus avaient réalisé l'apprentissage de l'autosondage avec une infirmière spécialisée et suivant un même protocole codifié d’ETP (éducation thérapeutique du patient), un mois auparavant.

Les critères d'inclusion étaient des patients de plus de 18 ans, réalisant les autosondages au moins une fois par jour. Les patients ayant appris les autosondages à titre préventif (injection de faible dose de toxine intra détrusorienne ou avant la pose d'une bandelette sous uréthrale) ont été exclus s'ils ne réalisaient aucun sondage au décours de I'apprentissage. Parmi les 86 patients, 9 ont été exclus en raison de données manquantes concernant la présence ou non d'une douleur ou bien sur les scores de symptômes étudiés. Au total 77 patients ont été inclus (Tableau 1).

Lors de la consultation de suivi un mois après l'apprentissage, les données concernant le nombre d'autosondages réalisés et les éventuelles difficultés à la réalisation du geste ont été recueillies. Pour avoir une évaluation objective de l'adhésion, de la satisfaction et des difficultés, les questionnaires ICAS [7], InCaSaQ [8] et ICDQ [9] étaient remplis par les patients.

La douleur aux autosondages était caractérisée par la réponse au score ICQD. Les résultats ont été binarisés : les patients répondant " un peu », " moyen » ou « beaucoup » à au moins une des 2 questions spécifiques portant sur l'existence de douleurs entraient dans la catégorie « douloureux » versus ceux ayant répondu « non » (" non douloureux »). Pour le score InCaSaQ, deux groupes ont été définis, ceux qui étaient " satisfaits » de la sonde qu'ils utilisaient (score $\geq 16$ ) versus ceux qui étaient « insatisfaits » (score $<16)$. Les résultats du score ICAS ont été divisés en 3 catégories : les patients ayant une forte adhésion (score $<1$ ), 
ceux ayant une adhésion moyenne (score entre 1 et 2,75) et ceux ayant une adhésion faible (score $\geq 3$ ).

Nous avons effectué une analyse univariée à l'aide d'un test de Fischer pour rechercher une association entre la douleur et les variables étudiées (âge, sexe, indice de masse corporelle (IMC), dyssynergie vésico sphinctérienne (DVS), type de sonde, score InCaSaQ, score ICAS et autosondages exclusifs ou non). La variable « âge » a été classée en 2 catégories (plus de 50 ans versus moins de 50 ans) et la variable «IMC » a été séparée en IMC $\leq 25 \mathrm{~kg} / \mathrm{m}^{2}$ versus IMC $>25 \mathrm{~kg} / \mathrm{m}^{2}$ afin de faciliter l'analyse statistique et l'interprétation des résultats. Nous avons réalisé une analyse multivariée par régression logistique en incluant les variables ayant un $p<0,2$ en analyse univariée.

Tous les résultats avec $p<0,05$ étaient considérés comme significatifs. Les statistiques ont été réalisées avec le logiciel $\mathrm{R}$-studio version 3.3.1.

Le consentement oral et écrit des patients a été obtenu ainsi que l'accord du CPP.

\section{RESULTATS}

Soixante-dix-sept patients ont été inclus (Tableau 1). L'âge moyen de la population était de 51,6 ans (sd 14,5) avec une population principalement atteinte de pathologies neurologiques centrales (tableau 1) dont 52/77 avaient une dyssynergie vésico sphinctérienne.

Tous ces patients avaient une sensibilité urétrale conservée, hormis les 6 patients souffrant d'un syndrome de la queue de cheval dont le niveau lésionnel déterminait une hypoesthésie urétrale.

D'après les réponses au questionnaire ICDQ, $28 / 77$ patients $(36,4 \%)$ se plaignaient d'une douleur lors de l'autosondage. Les autosondages étaient réalisés en moyenne 4,4 fois par jour (sd 1,8) et 38 patients $(49,4 \%)$ les réalisaient de manière exclusive. Les résultats de l’analyse univariée sont présentés dans le tableau 2. 
En analyse univariée, on retrouvait une association significative entre la douleur à l'autosondage et une faible adhésion au traitement $(p=0,01)$. L'analyse multivariée (Tableau 3) a inclu les variables avec $p<0,2$ en analyse univariée : sexe, score ICAS et IMC.

Ces douleurs étaient associées en analyse multivariée à une faible ou moyenne adhésion des patients à l'autosondage (OR 25,28 [3,72-172,03]) ou au sexe féminin (OR 3,99 [1,11-14,37]). En revanche, un IMC élevé (> 25kg/m2) semblait avoir un effet protecteur sur l'existence de douleurs lors de l'autosondages avec un OR 0,31 [0,31-0,99].

\section{DISCUSSION}

Dans cette étude, $36,4 \%$ des patients réalisant des autosondages depuis au moins un mois se plaignaient de douleurs lors de la réalisation du geste. Ces données démontrent une prévalence de douleurs lors du cathérisme intermittent légèrement supérieures à celles de la littérature. Dans un étude de 2011, par Kessler et al [4] chez 92 patients admis en service d'urologie, il était retrouvé 13/92 (14\%) des patients qui avaient une douleur modérée ou sévère lors du sondage. Cette différence peut être liée à une population différente, puisque recrutée dans un centre urologique pour Kessler et en neuro-urologie pour notre cohorte. Pour autant, notre population principalement neurologique centrale, peut présenter des anomalies sensitives avec hyperesthésie d'origine spinale ou corticale. De plus, dans l'étude de Kessler et al [4] l'évaluation de la douleur se faisait via une échelle visuelle analogique et non pas par un questionnaire validé (ICDQ) comme nous l'avons réalisé, pouvant ainsi rendre plus difficile l'interprétation des résultats. Dans notre étude, tous les patients utilisaient des sondes autolubrifiées. Ces sondes permettent a priori de réduire le risque de micro traumatismes et potentiellement de douleurs lors du sondage, en comparaison à une sonde sèche $[2,10]$. 
Parmi les différentes sondes utilisées par les patients, il n'existe pas de différence en termes de douleurs lors du sondage, en fonction de la marque de la sonde et de son calibre (ch). La relation directe que nous avons retrouvée entre douleur et adhérence aux autosondages (via le questionnaire I-CAS) est bien compréhensible. La douleur est d'une manière générale un facteur limitant d'activités, quelqu'en soit leur type, motrices, gestuelles, voire sociales. La crainte de la reproduction des symptômes douloureux par le geste d'auto-cathétérisme induit une tentative à se soustraire au traitement quelqu'en soit la légitimité, la nécessité et même le résultat en termes de bénéfices fonctionnels (disparition de fuites ou d'une pollakiurie) ou organiques (disparition des infections urinaires, protection du haut appareil).

Concernant la prévalence des douleurs suivant le sexe, on aurait pu s'attendre à une prévalence plus importante chez l'homme en raison de la longueur de l'urétre, de l'existence de coudures anatomiques et d'éventuelle hypertrophie prostatique associée. Ce n'est pas le cas probablement en raison de la difficulté pour nombre de femmes à repérer de manière immédiate le méat urétral et à faire progresser de manière anatomiquement axée, le cathéter lors du sondage.

La relation entre le BMI et l'existence de douleur, mériterait des analyses et des études complémentaires en raison des possibles difficultés d'accessibilité et de progression du cathéter chez les obèses, et de la possible existence d'un syndrome métabolique associé pouvant engendrer des micro-neuropathies distales avec hyperesthésie et hyperalgie de déafférentation. L'obésité reste un facteur de mauvais pronostique d'adhésion à la réalisation de l'autosondage où à un mois [12]. 
Notre étude comporte cependant un certain nombre de limites.

Le caractère rétrospectif en est une mais peu de données sont disponibles dans la littérature concernant la proportion de douleurs aux autosondages et les moyens mis en place pour les prendre en charge. L'impact de ces douleurs sur l'adhérence aux autosondages invite à proposer des solutions thérapeutiques spécifiques pour prévenir, supprimer ou réduire les douleurs per et post cathétérisme. Plusieurs traitements sont en effet possibles, tels que l'ajout d'alphabloquants, la réalisation d'injection de toxine botulinique intra sphinctérienne qui peut faciliter la réalisation des autosondages [11] , l'adjonction d'antalgiques neurotropes, le changement de matériel, de type d'embout.

Ces sensations de blocage ou les douleurs ressenties lors d'un geste qu'il convient de réaliser, en général, de manière pluriquotidienne, peut impacter la qualité de vie. C'est ce que retrouve Kessler et al [4] dans son étude, où une douleur sévère (EVA > 7) lors du sondage diminuait la qualité de vie. Cependant dans notre étude, la satisfaction liée à l'autosondage n'était pas associée à la présence ou non d'une douleur lors du cathérisme. De nouvelles études propsectives sont nécessaires afin d'évaluer l'impact du sexe et du BMI sur les douleurs liées aux autosondages. Il serait également intéressant d'étudier l'adhésion à l'autosondage un peu plus à distance, par exemple à 6 et 12 mois ou encore de vérifier si la douleur lors du sondage pourrait être un facteur d'échec à leur réalisation sur le long terme.

\section{CONCLUSION}

Dans une population de patients suivis pour des troubles vésico sphinctériens principalement liés à une pathologie neurologique, 36\% d'entre eux décrivaint des douleurs 
lors de la réalisation de l'autosondage. II semble exister une association entre la présence de douleurs et une adhésion faible à un mois de l'apprentissage de l'auto-cathétérisme. Une évaluation et une prise en charge spécifiques de ces douleurs sont à prendre en compte lors de l'introduction d'un traitement par autosondages, notamment dans une population de patients neurologiques, mais aussi tout au long du suivi.

\section{REFERENCES}

[1] Tornic J, Sartori AM, Gajewski JB, Cox A, Schneider MP, Youssef NA and al. Catheterization for treating neurogenic lower urinary tract dysfunction in patients with multiple sclerosis: A systematic review. A report from the Neuro-Urology Promotion Committee of the International Continence Society (ICS). Neurourol Urodyn. 2018;37(8):2315-2322.

[2] Chartier-Kastler E, Denys P. Intermittent catheterization with hydrophilic catheters as a treatment of chronic neurogenic urinary retention. Neurourol Urodyn. 2011;30(1):21-31. [3] Stoffel JT, Peterson AC, Sandhu JS, Suskind AM, Wei JT, Lightner DJ. AUA White Paper on Nonneurogenic Chronic Urinary Retention: Consensus Definition, Treatment Algorithm, and Outcome End Points. J Urol. 2017;198(1):153-160.

[4] Kessler TM, Ryu G, Burkhard FC. Clean intermittent self-catheterization: a burden for the patient? Neurourol Urodyn. 2009;28(1):18-21.

[5] Yılmaz B, Akkoç Y, Alaca R, Erhan B, Gündüz B, Yıldız N and al. Intermittent catheterization in patients with traumatic spinal cord injury: obstacles, worries, level of satisfaction. Spinal Cord. 2014;52(11):826-30. 
[6] Motavasseli D, Chesnel C, Charlanes A, Menoux D, Charoenwong F, Le Breton F, Amarenco G. Adherence to Anticholinergic Therapy and Clean Intermittent SelfCatheterization in Patients With Multiple Sclerosis. Int Neurourol J. 2018;22(2):133-141. [7] Guinet-Lacoste A, Charlanes A, Chesnel C, Blouet E, Tan E, Le Breton F, Amarenco G. Intermittent Catheterization Adherence Scale (ICAS): A new tool for the evaluation of patient adherence with clean intermittent self-catheterization. Neurourol Urodyn. $2018 ; 37(8): 2753$ 2757.

[8] Guinet-Lacoste A, Jousse M, Verollet D, Sheikh Ismael S, Le Breton F, Tan E and al. Validation of the InCaSaQ, a new tool for the evaluation of patient satisfaction with clean intermittent self-catheterization. Ann Phys Rehabil Med. 2014;57(3):159-68.

[9] Guinet-Lacoste A, Jousse M, Tan E, Caillebot M, Le Breton F, Amarenco G. Intermittent catheterization difficulty questionnaire (ICDQ): A new tool for the evaluation of patient difficulties with clean intermittent self-catheterization. Neurourol Urodyn. 2016;35(1):85-9.

[10] Stensballe J, Looms D, Nielsen PN, Tvede M. Hydrophilic-coated catheters for intermittent catheterisation reduce urethral micro trauma: a prospective, randomised, participant-blinded, crossover study of three different types of catheters. Eur Urol. 2005;48(6):978-83.

[11] Honore T, Le Breton F, Turmel N, Bignani B, Chesnel C, Charlanes A, Amarenco G. Efficacy of botulinum toxin A injections in the urethral sphincter in patients with difficulties to perform self-intermittent catherization. Prog Urol. 2018;28(7):370-376.

[12] Hentzen C, Haddad R, Ismael SS, Peyronnet B, Gamé X, Denys P and al. Predictive factors of adherence to urinary self-catheterization in older adults. Neurourol Urodyn. 2019;38(2):770-778. 
Tableau 1. Analyse de la douleur urétrale lors de la pratique de l'autosondage : population d'étude.

\begin{tabular}{|c|c|}
\hline Sexe & $\mathbf{N}(\%)$ \\
\hline Hommes & $23(29 \%)$ \\
\hline Femmes & $54(70 \%)$ \\
\hline \multicolumn{2}{|l|}{ Pathologies } \\
\hline SEP & $35(45 \%)$ \\
\hline Atteinte médullaire & $9(11 \%)$ \\
\hline Syndrome de la queue de cheval & $6(7 \%)$ \\
\hline Idiopathique & $6(7 \%)$ \\
\hline Multifactoriel & $4(5 \%)$ \\
\hline Autre & $17(22 \%)$ \\
\hline \multicolumn{2}{|l|}{ Type de sonde } \\
\hline Lofric & $29(37 \%)$ \\
\hline Speedicath & $34(44 \%)$ \\
\hline Vapro ou Infyna & $9(11 \%)$ \\
\hline Autre & $5(6 \%)$ \\
\hline Indice de masse corporelle (IMC), moyenne (écart type) & $24,1(\mathrm{sd} 5,5)$ \\
\hline$<25 \mathrm{~kg} / \mathrm{m}^{2}$ & $49(63,6 \%)$ \\
\hline$>25 \mathrm{~kg} / \mathrm{m}^{2}$ & $28(36,4 \%)$ \\
\hline Intermittent catheterization difficulty questionnaire (ICDQ): & $5,1(\mathrm{sd} 8,3)$ \\
\hline Intermittent Catheterization Adherence Scale (ICAS) & $1,9(\mathrm{sd} 1,7)$ \\
\hline Adhésion faible (score $>3$ ) & $24(31,1 \%)$ \\
\hline Adhésion moyenne (score entre 1 et 2 ) & $33(42,9 \%)$ \\
\hline Adhésion forte (score $<1)$ & $20(26 \%)$ \\
\hline Intermittent Catheterization Satisfaction Questionnaire (InCaSaQ) & $18,5(\mathrm{sd} 3,8)$ \\
\hline Satisfait (score $>16$ ) & $61(79 \%)$ \\
\hline Insatisfait (score < 16) & $16(20 \%)$ \\
\hline
\end{tabular}


Tableau 2. Analyse de la douleur urétrale lors de la pratique de l'autosondage : analyse univariée

\begin{tabular}{|c|c|c|}
\hline & DOULEURS & $\mathbf{p}$ \\
\hline Sexe & & 0,17 \\
\hline Hommes & $11 / 23(47 \%)$ & \\
\hline Femmes & $17 / 54(31 \%)$ & \\
\hline Age & & 0,40 \\
\hline Moins de 50 ans & $13 / 31(41 \%)$ & \\
\hline Plus de 50 ans & $15 / 46(32 \%)$ & \\
\hline Indice de masse corporelle (IMC) & & 0,12 \\
\hline$<25 \mathrm{~kg} / \mathrm{m}^{2}$ & $21 / 49(42 \%)$ & \\
\hline$>25 \mathrm{~kg} / \mathrm{m}^{2}$ & $7 / 28(25 \%)$ & \\
\hline Intermittent Catheterization Adherence Scale (ICAS) & & $0,01^{*}$ \\
\hline Adhésion faible & $13 / 24(54 \%)$ & \\
\hline Adhésion moyenne & $13 / 33(39 \%)$ & \\
\hline Adhésion forte & $2 / 20(10 \%)$ & \\
\hline $\begin{array}{l}\text { Intermittent Catheterization Satisfaction Questionnaire } \\
\text { (InCaSaQ) }\end{array}$ & & 0,21 \\
\hline Satisfait & 20/61 (32\%) & \\
\hline Insatisfait & $8 / 16(50 \%)$ & \\
\hline Type de sonde & & 0,56 \\
\hline Lofric & $9 / 29(31 \%)$ & \\
\hline Speedicath & $13 / 34(38 \%)$ & \\
\hline Vapro ou Infyna & $5 / 9(55 \%)$ & \\
\hline Autre & $1 / 5(20 \%)$ & \\
\hline Dyssynergie vésico sphinctérienne & & 0,29 \\
\hline Oui & $21 / 52(40 \%)$ & \\
\hline Non & $7 / 25(28 \%)$ & \\
\hline Autosondages & & 0,39 \\
\hline Exclusifs & $12 / 38(31 \%)$ & \\
\hline Associés à des mictions spontanées & $16 / 39(41 \%)$ & \\
\hline
\end{tabular}


Tableau 3. Analyse de la douleur urétrale lors de la pratique de l'autosondage : analyse multivariée

\begin{tabular}{|c|c|c|c|}
\hline & OR (IC 95\%) & OR ajusté (IC 95\%) & $\mathbf{p}$ \\
\hline \multicolumn{4}{|l|}{ IMC } \\
\hline \multicolumn{4}{|l|}{$<25 \mathrm{~kg} / \mathrm{m}^{2}$ (Ref) } \\
\hline$>25 \mathrm{~kg} / \mathrm{m}^{2}$ & $0,44[0,16-1,24]$ & $0,31[0,31-0,99]$ & $0,04^{*}$ \\
\hline \multicolumn{4}{|l|}{ Sexe } \\
\hline \multicolumn{4}{|l|}{ Masculin (Ref) } \\
\hline Féminin & $2,00[0,73-5,42]$ & $3,99[1,11-14,37]$ & $0,03^{*}$ \\
\hline \multicolumn{4}{|l|}{ ICAS } \\
\hline \multicolumn{4}{|l|}{ Adhésion forte (Ref) } \\
\hline Adhésion moyenne & $5,85[1,16-29,53]$ & $6,70[1,22-36,89]$ & $0,03^{*}$ \\
\hline Adhésion faible & $10,64[2,01-56,33]$ & $25,28[3,72-172,03]$ & $<0,01^{*}$ \\
\hline
\end{tabular}

Abréviations : IC, intervalle de confiance ; ICAS, Intermittent Catheterization Adherence Scale ; IMC, indice de masse corporelle ; OR, odds ratio ; Ref, référence. 